Vol.02/ No. 03

Pages: $123-128$

https://www.irojournals.com/iroeea

DOI: https://doi.org/10.36548/jeea.2020.3.003

\title{
Electric Vehicle Charging with Battery Scheduling and Multicriteria Optimization using Genetic Algorithm
}

\author{
Nayana, \\ Associate Professor, \\ Dept. of EE Engineering NMAMIT, \\ Nitte, Udupi, Karnataka. \\ Email id: nayanar401@gmail.com
}

\begin{abstract}
The existing charging infrastructure needs expansion and upgrade with the growing fleet of electric vehicles (EV). The electric grids are largely affected by the uncontrolled charging cycles. To overcome this drawback, the hybrid charging stations are incorporated with battery storage and renewable energy sources. The power necessary from the grid can be buffered using a battery and renewable source attached to the charging station thereby avoiding the grid constraints and peaks. It has been a challenge to trace the origin of the battery's energy till date. The battery energy storage and a simple photovoltaic system is incorporated in a hybrid EV charging station. Uncontrolled EV charging and its adverse effects can be overcome by this technology by accurately calculating the share of renewable energy derived from the battery. Multi-attribute utility theory is used for optimizing the EV charging level and scheduling the battery charging and discharging. Minimizing battery degradation and charging cost while maximizing the renewable energy from the battery and PV sources are the major criteria of optimization. Multicriteria optimization function is used along with the genetic algorithm optimization scheme to address the optimization issues. Optimal capacity of the battery and optimization strategy is affected by the preferences in decision making.
\end{abstract}

Keywords: Battery scheduling, multicriteria optimization, electric vehicle charging, genetic algorithm, energy optimization;

\section{Introduction}

There is a rapid growth in electric vehicles in the recent past due to the global energy policy, climate change and environmental pollution [1]. Various studies are conducted with regard to EV charging systems. Increased loss of energy, distribution network transformer overloading, battery temperature or voltage increase leading to decrease in the EV battery capacity or deterioration of the battery, power quality reduction due to harmonic distortion and voltage dips are the major drawbacks caused by lack of coordination in the EV chargers and charging schemes [2]. Energy is provided for EV charging using solar and other renewable energy sources due to their abundance and clean nature. Small EVs are integrated in the EV charging stations with photovoltaic (PV) generation modules using various control strategies while reducing the carbon footprint and lowering the cost.

Fuel cell vehicles, solar-charged EVs and plug-in hybrid electric vehicle (PHEV) are integrated with the residential photovoltaic system that is grid-connected using various strategies [3]. Wind generator and PV array are integrated with EV in several recent research models. The variability in production of PV is a significant drawback of charging EV with PV. Often, the battery energy storage is used for addressing the arbitrary nature of renewable energy [4]. The adverse effects like grid losses and peak load demand can be reduced with the combination of battery energy system and EV [5]. When excess of solar or wind energy is available, charging of energy storage is performed. EV is charged using the storage when there is insufficiency in generation [6]. The local storage charging and discharging can be optimized using various algorithms when used with EV chargers. The battery can be discharged throughout specific time of the days at a constant rate. The total connected EVs and available solar energy can be estimated. However, the total renewable energy available in the battery is not estimated [7].

Grid energy reduction can be done by around $25 \%$ while alleviating the variations in daily solar energy with a $10 \mathrm{kWh}$ small-sized energy storage model [9]. Fast charging units, lithium ion battery and PV system can be integrated into an EV station for fast charging operation and control. Minimum energy consumption from the grid is ensured in the fast charging stations with the help of appropriate control algorithm. Fast chargers with a charging current of $120 \mathrm{~A}$ are considered for analysis. Flexibility of the distribution network can be increased with the help of EV chargers with battery buffer. Ancillary services like voltage control, primary and secondary frequency control features can be provided for supporting the power grid [10]. The PV production is matched with the EV 
Journal of Electrical Engineering and Automation (EEA) (2020)

Vol.02/ No. 03

Pages: $123-128$

https://www.irojournals.com/iroeea

DOI: https://doi.org/10.36548/jeea.2020.3.003

charging with the flexibility provided by various smart charging schemes. Reduction of vehicle charging cost, ancillary service revenue maximization and service waiting time reduction can be implemented during the daily scheduling of EV fleet using multi-objective decision-making scheme. Queuing theory may be used for modelling the service waiting time and total client requests.

\section{Literature Review}

Several constraints and criteria are used in various control and optimization algorithms for determining the control scheme and optimal size of the battery energy system [11]. The customer's perspective of cost reduction and grid point of view of load variance reduction in the energy storage system is optimized. Real-time electricity cost can be used for reduction of operational cost. Combination of battery energy system or gridconnected PV can be used for developing a financial optimization model based on particle swarm optimization (PSO). The power imported from the grid can be minimized while optimizing the power installed from storage and renewable energies and reducing the number of chargers using genetic algorithm while designing EV fastcharging station. Grid connection is not considered in certain literature where installation of PV and EV chargers are done in individual houses for smart charging [12].

The battery operation is scheduled ahead of a day in most of the literature. However, optimization ahead of an hour is preferred in real-time control. The total operational cost can be decreased by integration of commercial building, fixed battery storage and PV to the charging station using control algorithm and four-stage optimization [13]. Profit from selling excess power back to the grid, charging and discharging cost of EVs, unsupplied demand cost, PV and BES operational cost, and the distribution grid's power cost are the elements of the total cost of operation. The uncertainties of optimization parameters can be overcome by implementing chance-constrained optimization scheme. The source of energy for charging and discharging the battery is not considered despite the high tolerance provided by the model towards uncertain parameters. Accurate amount of renewable energy required for charging the $\mathrm{EV}$ is also not considered despite several factors involved in current optimization schemes [7]. Single-criterion optimization is another significant drawback of the existing research schemes. The objective function consists of factors like profit of charging station owners, load loss, customer satisfaction, and operational cost. The previous research work does not estimate the accurate amount of renewable energy used in the process of EV charging despite proposing various optimization schemes. Multicriteria approach is also not available in the current research schemes which is a major drawback of the existing systems [12]. The objective function consists the factors associated with the owners of the charging stations like profit, load loss, customer satisfaction and operational cost [14].

\section{Proposed Work}

The origin of EV charging energy can be tracked and uncontrolled EV charging effects can be eliminated using the model proposed in this paper. Multi-attribute utility theory is used for optimization of the charging levels of EV and the battery charging and discharging is scheduled using multicriteria optimization scheme [3]. Battery degradation is minimized along with the charging cost, while renewable energy share is maximized in this optimization criteria. The various conflicting criteria can be simultaneously optimized using this multi-objective technique. It is also possible to track the origin of energy and monitor its flow to and from the battery. The EV charging power and BES charging and discharging can be optimized simultaneously using this method. The multicriteria optimization function is implemented using genetic algorithm.

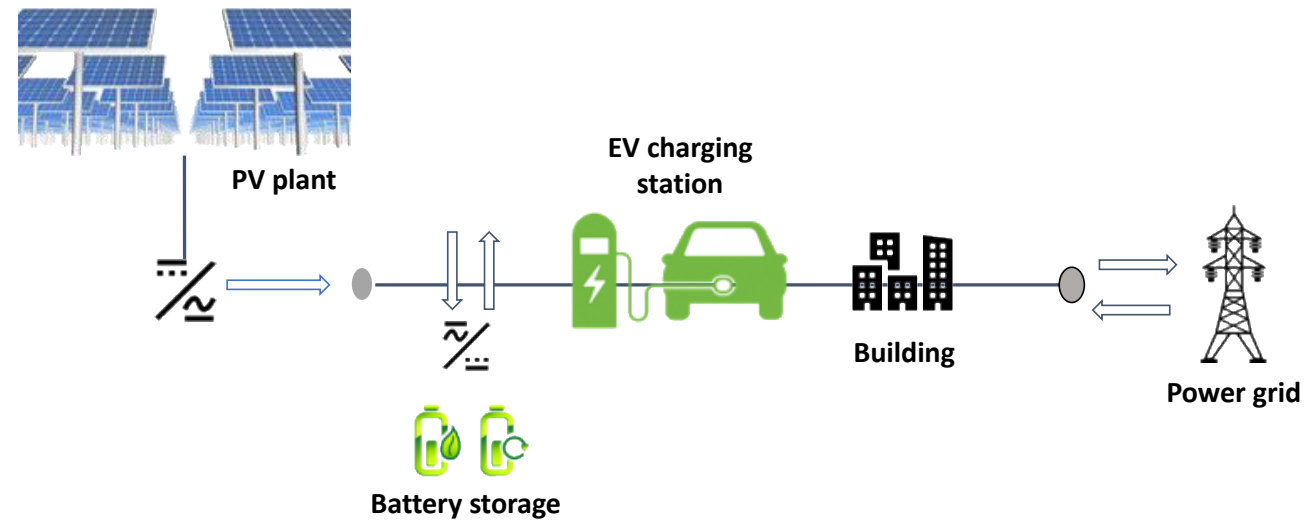

Figure 1: Electric Vehicle Charging Structure

ISSN: 2582-3051 (online)

Submitted: 16.12.2020

Accepted: 13.01.2021

Published: 19.01.2021 
Journal of Electrical Engineering and Automation (EEA) (2020)

Vol.02/ No. 03

Pages: 123-128

https://www.irojournals.com/iroeea

DOI: https://doi.org/10.36548/jeea.2020.3.003

There are several ways for charging electric vehicles through low voltage $\mathrm{AC}$ lines. It can be done from battery, network or from PV power plant. Charging can be performed from the battery in the discharge mode when it is not charging. The vehicle charging and individual system load can be covered with just the PV panels if they produce enough energy. If the energy produced by the PV panels is insufficient, only the network can be used for charging. Figure 1 represents the principle electric vehicle charging structure. Here the energy storage system and solar power plant of a public distribution network is connected to an EV for the individual charging system [8]. The direction of power is indicated by the arrows. The source of energy must be monitored as it is a significant criteria similar to renewable source energy maximization. The instantaneous power and network state determines the total energy that charges a battery. The network power flow equations can be solved for determining the value of this energy. The battery capacity limits the maximum charging energy. The mediumsized energy storage systems use lithium ion batteries most commonly due to their degradation model. With cycling and calendar aging, the storage capacity of these batteries reduce. The short term planning horizon is not affected by calendar aging. The energy throughput, rate of charging and discharging as well as temperature affects the cycle life loss. An exponential function is used for capturing the cycling induced battery cell capacity loss and discharge rate effects.

\section{Results and Discussion}

The operation of the hybrid system is analysed across various aspects like battery power and grid power with respect to variation in time. Figure 1 represents the battery schedule for charging and discharging. Other factors like EV charging power, battery state of charge and power flow is also analysed. In this proposed model we use a battery with $\mathrm{C}=50 \mathrm{kWh}$ capacity is considered. A $22 \mathrm{~kW}$ power rated $\mathrm{EV}$ charge and a $100 \mathrm{~kW}$ power rated solar power plant is also incorporated in the system. Over a duration of 6 to 12 hours, $60 \mathrm{kWh}$ charging and over 16 to 22 hours, $100 \mathrm{kWh}$ charging of the EV is obtained.

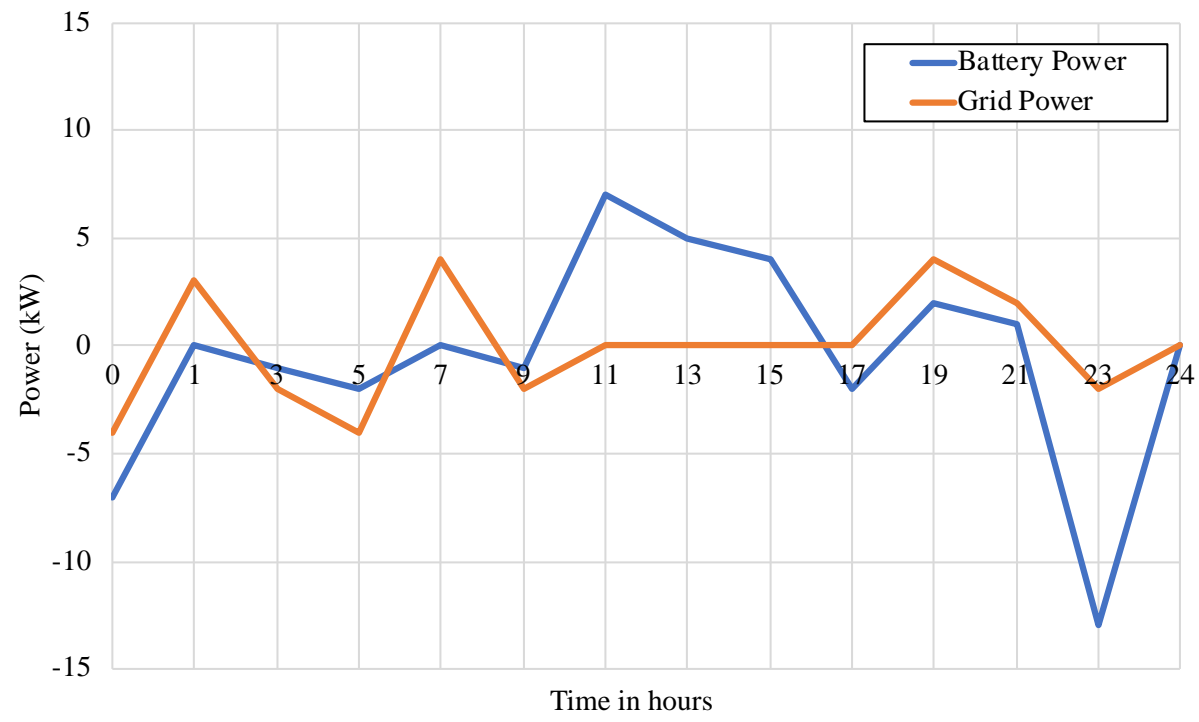

Figure 2: Battery schedule for charging and discharging

Figure 3 represents the charging power of the EV during multicriteria optimization. Factors like battery schedule for charging and discharging, battery SOC and power flows are also analysed during multicriteria optimization. Common weighting factor is considered for each criteria in one iteration. The next iteration considers multiple weighting coefficients for performing the optimization function. Charging an electric vehicle using renewable energy is given higher priority in additive model, whereas, the criteria values are distributed in a balanced manner in complementary case. Reactive power generation is not considered for the sake of simplification. Unity power factor mode is selected for the functioning of all inverters. In the initial stage, each criterion is analysed and the corresponding utility function is determined. Further, the second stage, the EV charging and battery scheduling is done using multi-attribute approach. The battery cost is also not considered as the battery capacity is maintained constant for all simulation.

The PV power plant and public grid provides the energy required for charging the battery. We offer a framework to monitor the renewable energy that enters and leaves the battery thereby providing information 
Journal of Electrical Engineering and Automation (EEA) (2020)

Vol.02/ No. 03

Pages: $123-128$

https://www.irojournals.com/iroeea

DOI: https://doi.org/10.36548/jeea.2020.3.003

regarding the amount of renewable energy utilized for charging the EV. The instantaneous power of the sources involved and the network state act as the deciding factor of the energy entering the system. The network power flow equations are solved. The battery capacity limits the maximal energy for charging the battery. During discharge operation, the relative ratio of energy available in various sources is used for determining the quantity of energy obtained from the battery.

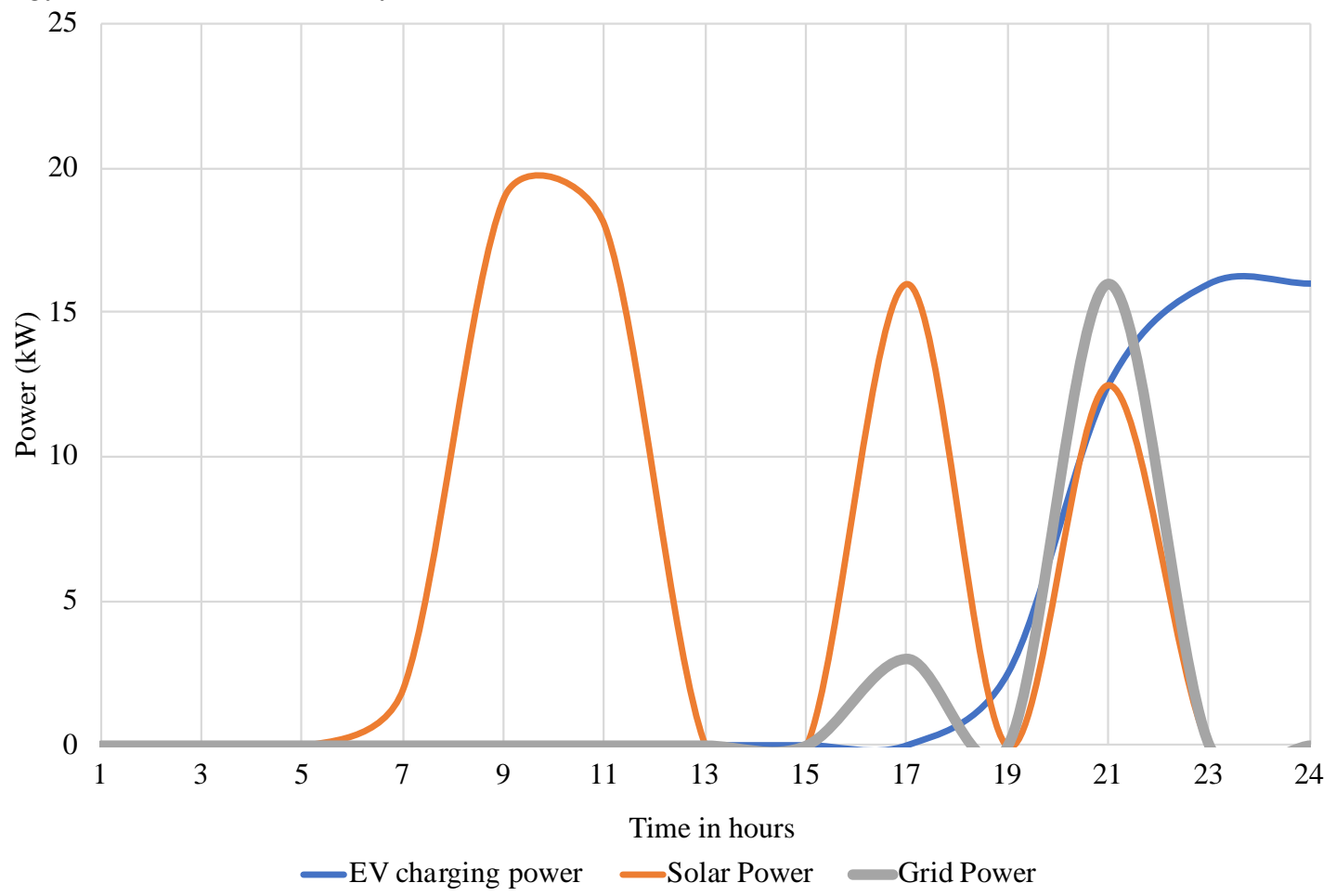

Figure 3: Charging power of EV across time

Figure 4 represents the dependence of utility function on the battery capacity. Power from the battery, public grid and PV are used for charging the EV. The major components of power can be categorized as battery power obtained from the grid, PV panel based battery power, public grid based power and PV panel power. The sum of these elements give the total power. Hourly battery charging and discharging is scheduled and optimized. The criteria considered in the optimization problem are renewable energy maximization, energy supply, operation and battery installation cost minimization as well as the battery aging factor. The multi-attribute utility theory (MAUT) indicates various conditions and functions to be met under multilinear, additive and multiplicative operations. Various interactions among individual criteria are reflected by the utility function. Balanced charging of the vehicle is performed without reaching the maximum allowable value. The total network load and individual load is proportional to the total solar energy. Due to this reason, the load of consumers in the station is directly affected by the total energy stored in the battery. 
Journal of Electrical Engineering and Automation (EEA) (2020)

Vol.02/ No. 03

Pages: $123-128$

https://www.irojournals.com/iroeea

DOI: https://doi.org/10.36548/jeea.2020.3.003

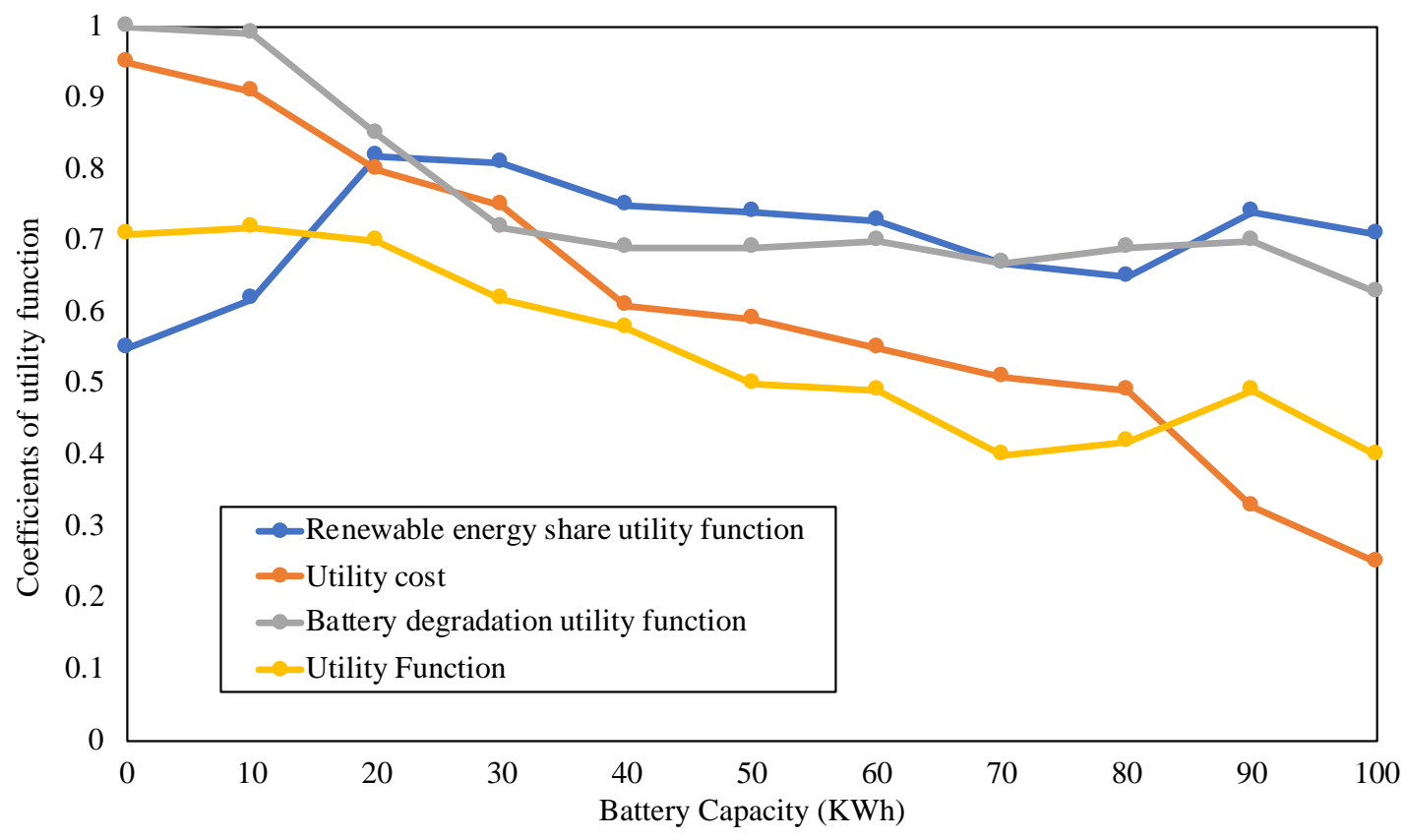

Figure 4: Dependence of utility function on battery capacity

\section{Conclusion}

A genetic algorithm scheme is proposed for scheduling the charging or discharging of an electric vehicle in a real time scenario using multicriteria optimization scheme in this paper. The share of renewable energy required for charging of EV is maximized by accurate tracking and monitoring the renewable energy on a real time basis. The total system cost inclusive of cost involved in importing energy from the grid, operational cost and battery installation cost is also optimized simultaneously along with the degradation rate of the battery. Quantification of the impact of various criteria on the problem's optimal solution is shown with the help of a hybrid system during simulation. The solar power plant provides over $85 \%$ of the renewable energy for charging EVs. Over $50 \%$ of the renewable energy is reduced during charging along with reduction in battery degradation during the process of charging or discharging while implementing multicriteria optimization. The preferences of various decision makers and their influence is quantified. The battery capacity can be further optimized using additive attribute aggregation. The battery degradation and system cost are analysed for monitoring the performance of the battery based on which the values are balanced.

\section{References}

[1] Petrusic, A., \& Janjic, A. (2021). Renewable Energy Tracking and Optimization in a Hybrid Electric Vehicle Charging Station. Applied Sciences, 11(1), 245.

[2] Abedinia, O., Lu, M., \& Bagheri, M. (2019). An improved multicriteria optimization method for solving the electric vehicles planning issue in smart grids via green energy sources. IEEE Access, 8, 3465-3481.

[3] Han, X., Liang, D., \& Wang, H. (2020). An optimization scheduling method of electric vehicle virtual energy storage to track planned output based on multiobjective optimization. International Journal of Energy Research, 44(11), 8492-8512.

[4] Chen, C., \& Zhang, G. (2019, October). Impact of large-scale electric vehicle charging on a power grid and its optimal scheduling strategy. In Emerging Developments in the Power and Energy Industry: Proceedings of the 11th Asia-Pacific Power and Energy Engineering Conference (APPEEC 2019), April 19-21, 2019, Xiamen, China (p. 61). CRC Press.

[5] Gampa, S. R., Jasthi, K., Goli, P., Das, D., \& Bansal, R. C. (2020). Grasshopper optimization algorithm based two stage fuzzy multiobjective approach for optimum sizing and placement of distributed generations, shunt capacitors and electric vehicle charging stations. Journal of Energy Storage, 27, 101117.

[6] Wei, H., Liang, J., Li, C., \& Zhang, Y. (2020). Real-Time Locally Optimal Schedule for Electric Vehicle Load via Diversity-Maximization NSGA-II. Journal of Modern Power Systems and Clean Energy.

[7] Na, Z., \& Zhong, T. (2018). Multi-objective optimization strategy for cooperative scheduling of electric vehicles and wind farms under V2G. Electrical Measurement \& Instrumentation, (12), 9. 
Journal of Electrical Engineering and Automation (EEA) (2020)

Vol.02/ No. 03

Pages: $123-128$

https://www.irojournals.com/iroeea

DOI: https://doi.org/10.36548/jeea.2020.3.003

[8] Sun, B. (2020). A multi-objective optimization model for fast electric vehicle charging stations with wind, PV power and energy storage. Journal of Cleaner Production, 125564.

[9] Zhang, L., Hu, X., Wang, Z., Sun, F., Deng, J., \& Dorrell, D. G. (2017). Multiobjective optimal sizing of hybrid energy storage system for electric vehicles. IEEE Transactions on Vehicular Technology, 67(2), 1027-1035.

[10] Garcia-Guarin, J., Infante, W., Ma, J., Alvarez, D., \& Rivera, S. (2020). Optimal scheduling of smart microgrids considering electric vehicle battery swapping stations. International Journal of Electrical and Computer Engineering, 10(5), 5093.

[11] Milas, N. T., Mourtzis, D. A., Giotakos, P. I., \& Tatakis, E. C. (2020, September). Two-Layer Genetic Algorithm for the Charge Scheduling of Electric Vehicles. In 2020 22nd European Conference on Power Electronics and Applications (EPE'20 ECCE Europe) (pp. P-1). IEEE.

[12] Mugunthan, S., \& Vijayakumar, T. (2019). Review on IoT based smart grid architecture implementations. j Electric Eng Autom, 1(1), 12-20.

[13] Bindhu, V. (2020). An Enhanced Safety System for Auto Mode E-Vehicles through Mind Wave Feedback. Journal of Information Technology, 2(03), 144-150.

[14] Rahimunnisa, K. (2019). Hybrdized Genetic-Simulated Annealing Algorithm for Performance Optimization in Wireless Adhoc Network. Journal of Soft Computing Paradigm (JSCP), 1(01), 1-13. 